\title{
Altmetric: $\mathrm{O}$ uso das mídias on-line para disseminar os artigos científicos
}

Amanda Costa Araujo ${ }^{1}$

${ }^{1}$ Programa de Mestrado Profissional em Inovação do Ensino Superior em Saúde, Universidade Municipal de São Caetano do Sul-USCS, Brasil.

A Prática Baseada em Evidência (PBE) consiste na tríade: melhor evidência disponível, preferências do paciente e experiência do profissional ${ }^{1}$. No entanto, o grande problema das pesquisas científicas é não atingir com grande proporção o público-alvo para quem o artigo científico é direcionado ${ }^{2,3,4}$. Nesse caminho, há uma lacuna entre o artigo científico e os leitores ${ }^{2}$.

Dentre os motivos para a falta de adesão a PBE estão: a falta de tempo para ler artigos científicos, a falta de acesso ao conteúdo, a dificuldade de encontrar artigos científicos e a língua de publicação ${ }^{1}$.

Por outro lado, atualmente as mídias sociais têm importante papel na vida das pessoas, seja para o trabalho ou para entretenimento ${ }^{5}$. A divulgação de posts na internet atinge grande público, seja para leitura ou compartilhamento de conteúdo ${ }^{5,6,7}$. Dessa forma, por que não usar as mídias sociais para divulgar artigos científicos?

Para esse objetivo a ferramenta Altmetric foi criada afim de, quantificar a visibilidade dos artigos científicos ${ }^{8}$. O Altmetric possui o formato de donut em que cada cor representa a menção por uma diferente mídia on-line (Figura 1$)^{8}$.

\section{Colors of the donut}

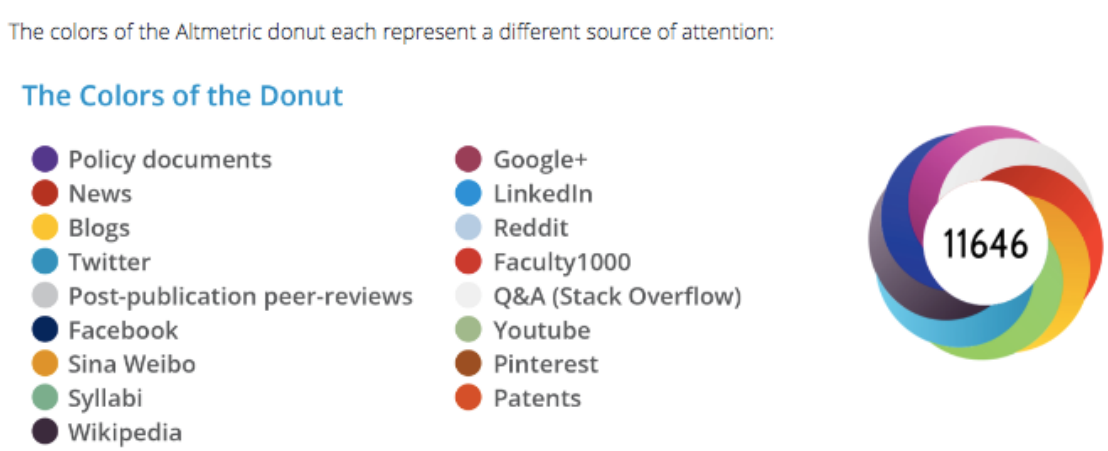

Figura 1. Representação das menções do Altmetric.

Por meio do Altmetric, o artigo científico é facilmente acessado, discutido e consequentemente mais divulgado ${ }^{9,10}$. Desta forma, pode abranger o público-alvo em larga escala. Uma vez postados, os artigos científicos podem ser disponibilizados na integra, o tema pode ser discutido por profissionais da área e compartilhados por uma grande rede de profissionais ${ }^{2,3,4}$. Além de que, o pesquisador consegue quantificar em quais mídias on-line o artigo está sendo visualizado e em quais países está sendo comentado (Figura 2$)^{8}$. 


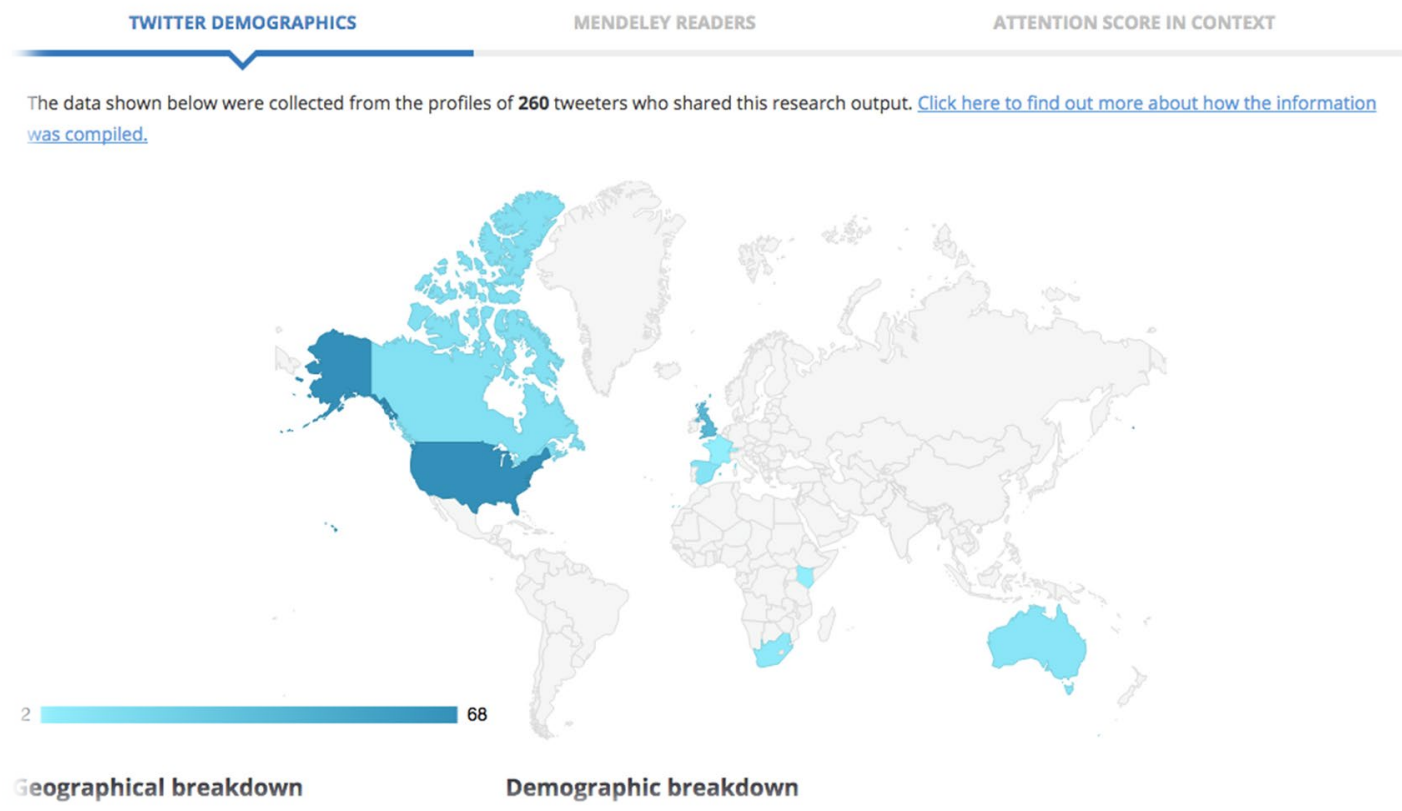

Figura 2. Representação da divulgação por países do Altmetric.

Para aumentar a visibilidade dos artigos científicos Araujo et al. ${ }^{3,4}$ recomenda que os artigos científicos sejam preferencialmente publicados em revistas com alto fator de impacto, tenham títulos provocativos (que demonstram os resultados do estudo no título) ou títulos interrogativos ${ }^{11}$. Além disso, recomenda-se que os artigos científicos sejam divulgados em mídias sociais, blogs e sites. Para a correta quantificação do Altmetric é necessário que o site contenha o DOI (Digital Object Identifier) do artigo científico ${ }^{8}$. Essas estratégias simples são importantes para aumentar a visibilidade dos artigos científicos. Além disso, fazer com o que a evidência científica abranja o público alvo ${ }^{12,13}$ e consequentemente a PBE seja utilizada de maneira efetiva ${ }^{2}$.

\section{Referências}

1. Herbert R, Jamtvedt G, Mead J, Hagen K. Practical Evidence-Based Physiotherapy. 2nd ed. Elsevier Butterworth-Heinemann 2011.

2. Araujo AC, Nascimento DP, Gonzalez GZ, Oliveira L, Costa P. How to increase the visibility of scientific articles through social media? Braz J Phys Ther. 2018;22(6):435436. doi:10.1016/j.bjpt.2018.08.009.

3. Araujo AC, Nascimento DP, Gonzalez GZ, Maher CG, Costa LOP. Impact of Low Back Pain Clinical Trials Measured by the Altmetric Score: Cross-Sectional Study. J Med Internet Res. 2018;20(4):1-9. doi:10.2196/jmir.9368.

4. Araujo AC, Gonzalez GZ, Nascimento DP, Costa LO. The impact of low back pain systematic reviews and clinical practice guidelines measured by the Altmetric score: Cross-Sectional Study. Braz J Phys Ther. 2020:Under review.

5. Dinsmore A, Allen L, Dolby K. Alternative Perspectives on Impact: The Potential of ALMs and Altmetrics to Inform Funders about Research Impact. PLOS Biol. 2014;12(11):1-4. doi:10.1371/journal.pbio.1002003.

6. Pinho-Costa L, Yakubu K, Hoedebecke K, et al. Healthcare hashtag index development: Identifying global impact in social media. J Biomed Inform. 2016;63(1):390-399. doi:10.1016/j.jbi.2016.09.010.

7. Patthi B, Prasad M, Gupta R, Singla A. Altmetrics - A Collated Adjunct Beyond Citations for Scholarly Impact: A Systematic Review. J Clin Diagnostic Res. 2017;11(6):16-20. doi:10.7860/JCDR/2017/26153.10078. 
8. Altmetric. Altmetric for Scopus. http://support.altmetric.com/knowledgebase/articles/83246-altmetric-for-scopus. Accessed December 19, 2019.

9. Rosenkrantz AB, Ayoola A, Singh K, Jr RD. Alternative Metrics ("Altmetrics") for Assessing Article Impact in Popular General Radiology Journals. Acad Radiol. 2017;24(7):1-7. doi:10.1016/j.acra.2016.11.019.

10. Berry M, Brunner N, Popescu S, Shukla P. Can apparent superluminal neutrino speeds be explained as a quantum weak measurement? J Phys A Math Theor. 2011;44(49):1-5. doi:10.1088/1751-8113/44/49/492001.

11. Deng B. Papers with shorter titles get more citations. Nature. 2017;2(8):1-4. doi:10.1038/nature.2015.18246.

12. Callaway E. Publishing elite turns against impact factor. Nature. 2016;535(7611):210211. doi:10.1038/nature.2016.20224.

13. Noone K. Beware the impact factor. Nature. 2013;45(5):513-515. doi:10.1038/nmat3566.

\section{Como citar este editorial:}

ARAUJO AC. Altmetric: O uso das mídias on-line para disseminar os artigos científicos. Rev. Aten. Saúde. 2021; 19(67): 3-5. 